\title{
Proving Finitely Presented Groups are Large by Computer
}

\author{
J. O. Button
}

\section{CONTENTS}

\section{Introduction}

2. Description of the Algorithm

3. Two-Generator One-Relator Presentations

4. Closed Hyperbolic 3-Manifolds

References
2000 AMS Subject Classification: 20-04

Keywords: finitely presented, large
We present a theoretical algorithm that given any finite presentation of a group as input, will terminate with answer yes if and only if the group is large. We then implement a practical version of this algorithm using MAGMA and apply it to a range of presentations. Our main focus is on two-generator one-relator presentations, for which we have a complete picture of largeness if the relator has exponent sum zero in one generator and word length at most 12, as well as if the relator is in the commutator subgroup and has word length at most 18. Indeed, all but a tiny number of presentations define large groups. Finally, we look at fundamental groups of closed hyperbolic 3-manifolds, for which the algorithm readily determines that at least a quarter of the groups in the SnapPea closed census are large.

\section{INTRODUCTION}

In this paper all groups considered will be finitely presented.

Definition 1.1. A finitely presented group $G$ is said to be large if $G$ has a finite-index subgroup that possesses a surjective homomorphism to a nonabelian free group.

This is a strong property, which implies a whole host of consequences: the most relevant one here is that $G$ has infinite virtual first Betti number (meaning that $G$ has finite-index subgroups with arbitrarily large first Betti number).

Perhaps surprisingly, it is currently unknown whether there is an algorithm that takes as input a finite presentation and tells us whether the group defined by that presentation is large. The two standard methods used in establishing unsolvability are to show that the property is Markov or is incompatible with free products (see [Lyndon and Schupp 77, Section 4.4]), but the property of being large is neither of these, and nor is its negation. However, there is a partial algorithm for largeness, as 
pointed out by I. Kapovich, that is guaranteed to terminate with the answer yes if the input presentation gives rise to a large group but that will not terminate otherwise. In [Holt and Rees 96], it is noted that there is a partial algorithm that will tell whether a presentation has a free quotient of rank 2 (but which will not terminate otherwise). Therefore, one can begin running this on a given finite presentation for a group $G$. Although this might run forever, even if $G$ is large, one immediately starts elsewhere a separate new process to evaluate the finite-index subgroups of $G$, one index at a time (using, say, the Todd-Coxeter or the Knuth-Bendix algorithm) along with a presentation for each subgroup, and then further starts the free-quotient algorithm many times in parallel on each finite-index subgroup. But nobody would ever want to implement this: the free-quotient algorithm is described in [Holt and Rees 96] as being totally impractical for long presentations, so we would be running an extremely slow process a vast number of times simultaneously.

In this paper we describe an alternative partial algorithm for largeness that one might actually want to implement. Furthermore, we do this by writing a program in MAGMA applying it to a considerable range of presentations. The theoretical result that allows us to establish largeness of a given finitely presented group $G$ is [Button 09, Theorem 2.1], which, in turn, is based on a result in [Howie 98]. It says that if $G$ is a finitely presented group having a homomorphism $\chi$ onto $\mathbb{Z}$ such that the Alexander polynomial $\Delta_{G, \chi}$ is zero, or zero modulo a prime $p$, then $G$ is large. The Alexander polynomial is an element (up to units) of the Laurent polynomial ring $\mathbb{Z}\left[t^{ \pm 1}\right]$ and will be defined in Section 2, where it is also shown in Proposition 2.1 that a large group will have a finite-index subgroup with a zero Alexander polynomial. It can be calculated reasonably efficiently given a presentation for $G$ (the time-consuming part of the process for long presentations being the calculation of large determinants), so it would seem that one needs to go through the finite-index subgroups $H$ and check each Alexander polynomial in turn.

That is the idea, but the problem is that when $\beta_{1}(H) \geq 2$, we have infinitely many homomorphisms from $H$ onto $\mathbb{Z}$. Therefore we need to establish that we can determine by a finite process whether there exists one of these homomorphisms with zero Alexander polynomial. Indeed, it will often be a finite-index subgroup of $G$ with first Betti number at least two that allows us to conclude largeness, so we will want to be able to do this quickly.
We describe our partial algorithm in Section 2, first theoretically, where we show that if the input is a finite presentation for a large group, then the algorithm is guaranteed in principle to prove largeness. We then describe how it was implemented in practice on a computer.

In the rest of the paper we report our results in running it. Our first application in Section 3 is to groups with a presentation of deficiency 1 , that is, groups possessing a finite presentation that has one more generator than relator. We have a particular interest in establishing largeness of these groups: although they are not always large, unlike groups of deficiency two or above, we have results in [Button 09] and [Button 10] that they are often large. Therefore, it would be good to have experimental evidence of this as well. Moreover, if $G$ is a group with a deficiency-1 presentation, then on using the Reidemeister-Schreier rewriting process to obtain a presentation for a given finite-index subgroup $H$ of $G$, we find that the resulting presentation for $H$ also has deficiency 1 .

In Section 3, we consider only two-generator onerelator presentations. This is not because of any limitations of the method (indeed, in applying the program to such a presentation, each finite-index subgroup that we work with will have a deficiency-1 presentation as mentioned above, but this will rarely be a one-relator presentation) but because we are able to cover a lot of ground in this special case.

We look at presentations for which one of the two generators has zero exponent sum in the relator, since any two-generator one-relator presentation can be put in such a form using an automorphism of $F_{2}$. For these presentations in which the relator is of length 12 or less, we have a definitive result. It can be summarized by saying that the vast majority of presentations are large, the presentations that are not large are listed in Table 1, and furthermore, all of these are groups that were already known not to be large. Indeed, they are all either Baumslag-Solitar groups or HNN extensions with base a Baumslag-Solitar group and cyclic associated subgroups. Sometimes the Baumslag-Solitar groups that appear are not presented in the standard way, but even these nonstandard presentations that occur were already known [McCool and Pietrowski 71] to define Baumslag-Solitar groups.

Among the presentations $\langle a, t \mid r\rangle$, where $r$ has exponent sum 0 in $t$, we have those for which the relator is of height 1 , which means that $r$ can be written purely in terms of $a^{ \pm 1}$ and $t a^{ \pm 1} t^{-1}$. We have a result in [Button 10] that gives a much more efficient criterion for largeness of 


\begin{tabular}{|c|c|c|c|c|}
\hline Length & Number & Presentation & Description & Large \\
\hline \multirow[t]{3}{*}{4.9} & 1 & $t a^{2}$ TataTa & $\mathrm{BS}(2,-3)$ & $\mathrm{x}$ \\
\hline & 2 & $t a^{2} T a t A T A$ & $D(1,1,2)$ & $\mathrm{x}$ \\
\hline & 3 & $t a^{2} T A t a T A$ & $\mathrm{BS}(2,3)$ & $\mathrm{x}$ \\
\hline \multirow[t]{10}{*}{4.11} & 4 & $t a^{2} T a^{2} t a^{2} T a$ & $\mathrm{BS}(3,-4)$ & $\mathrm{x}$ \\
\hline & 5 & $t a^{2} T a^{2} t a T a^{2}$ & $\cong \# 4$ & $\mathrm{x}$ \\
\hline & 6 & $t a^{2} T a^{2} t A T A^{2}$ & $D(2,1,2) \cong D(1,1,2)$ & $\mathrm{x}$ \\
\hline & 7 & $t a^{2} T a^{2} t A^{2} T A$ & $\cong \# 6$ & $\mathrm{x}$ \\
\hline & 8 & $t a^{2} T a t A^{2} T A^{2}$ & $\cong \# 6$ & $\mathrm{x}$ \\
\hline & 9 & $t a^{2} T A t a^{2} T A^{2}$ & $\mathrm{BS}(3,4)$ & $\mathrm{x}$ \\
\hline & 10 & $t a^{2} T A^{2} t a T A^{2}$ & $\cong \# 9$ & $\mathrm{x}$ \\
\hline & 11 & $t a^{3} T a t a^{2} T a$ & $\mathrm{BS}(2,-5)$ & $\mathrm{x}$ \\
\hline & 12 & $t a^{3} T a t A^{2} T A$ & $D(1,2,3)$ & $\mathrm{x}$ \\
\hline & 13 & $t a^{3} T A t a^{2} T A$ & $\mathrm{BS}(2,5)$ & $\mathrm{x}$ \\
\hline \multirow[t]{10}{*}{4.13} & 14 & $t a^{3} T a^{2} t a^{3} T A$ & & $\checkmark$ \\
\hline & 15 & $t a^{3} T a^{2} t a^{2} T a^{2}$ & $\mathrm{BS}(4,-5)$ & $\mathrm{x}$ \\
\hline & 16 & $t a^{3} T a^{2} t A^{2} T A^{2}$ & $D(2,2,3)$ & $\mathrm{x}$ \\
\hline & 17 & $t a^{3} T a^{2} t A^{3} T A$ & $D(3,2,1)$ & $\mathrm{x}$ \\
\hline & 18 & $t a^{3} T a t a^{3} T A^{2}$ & & $\checkmark$ \\
\hline & 19 & $t a^{3} T a t A^{3} T A^{2}$ & $\cong \# 17$ & $\mathrm{x}$ \\
\hline & 20 & $t a^{3} T A^{2} t a^{2} T A^{2}$ & $\mathrm{BS}(4,5)$ & $\mathrm{x}$ \\
\hline & 21 & $t a^{4} T a t a^{3} T a$ & $\mathrm{BS}(2,-7)$ & $\mathrm{x}$ \\
\hline & 22 & $t a^{4} T a t A^{3} T A$ & $D(1,3,4)$ & $\mathrm{x}$ \\
\hline & 23 & $t a^{4} T A t a^{3} T A$ & $\mathrm{BS}(2,7)$ & $\mathrm{x}$ \\
\hline \multirow[t]{4}{*}{6.13} & 24 & ta $a^{2}$ TataTataTa & $\mathrm{BS}(3,-4)$ & $\mathrm{x}$ \\
\hline & 25 & $t a^{2}$ TatATatATA & $?$ & $?$ \\
\hline & 26 & $t a^{2}$ TatAT AtATA & $?$ & $?$ \\
\hline & 27 & $t a^{2} T$ AtaT AtaT A & $\mathrm{BS}(3,4)$ & $\mathrm{x}$ \\
\hline \multirow[t]{2}{*}{6.14} & 28 & $t a^{2}$ Tata $a^{2}$ TataTa & $\mathrm{BS}(3,-5)$ & $\mathrm{x}$ \\
\hline & 29 & $t a^{2} T A t a^{2} T A t a T A$ & $\mathrm{BS}(3,5)$ & $\mathrm{x}$ \\
\hline
\end{tabular}

TABLE 1. Two-generator one-relator height-1 presentations.

such a presentation (involving only the abelianization of finite-index subgroups), and so we are able to consider height-one relators of length at most 14 . For these we are able to list all of the nonlarge presentations except for two, which we believe are not large yet we do not recognize them as groups that are already known not to be large.

Also in [Button 10] we gave an example of a large word-hyperbolic group that is of the form $F_{k} \rtimes_{\alpha} \mathbb{Z}$, but the automorphism $\alpha$ is reducible. Here we are able using the computer to give the first example of a large word-hyperbolic group of this form for which all nontrivial powers of the automorphism are irreducible.
We pay further attention to where the relator is in the commutator subgroup $F_{2}^{\prime}$. Until [Baumslag et al. 07], the only presentation known of this form that gave rise to a group that is not large is the case of $\mathbb{Z} \times \mathbb{Z}$ (for which the relator, if cyclically reduced, is just a commutator of the two generators). However, in that paper an example was given of a nonlarge group for which the particular relator $r_{0}$ is cyclically reduced and has length 18 . We again have a definitive result in that if $r$ is a cyclically reduced word in $F_{2}^{\prime}$ and has length at most 18, then our program shows that either the group $G$ is large, or $r$ has length 4 , so that $G=\mathbb{Z} \times \mathbb{Z}$, or $r$ has length exactly 18 and is just a cyclic permutation of $r_{0}$ or $r_{0}^{-1}$. 
Our final application, in Section 4, is to a class of groups that do not have deficiency-1 presentations but that always possess presentations of deficiency 0 . These are the fundamental groups of closed orientable hyperbolic 3-manifolds, and an open question asks whether they are always large. We already have a sample of over 10,000 examples to work with, which is the census from the program SnapPea [Hodgson and Weeks 01], and it is also available as a MAGMA database in which the fundamental groups of these 3 -manifolds are given.

It is known by recent results that if a closed hyperbolic 3-manifold is arithmetic and has positive virtual first Betti number, then we have largeness. However, positive virtual first Betti number for all 3-manifolds in the census was established using computational methods in [Dunfield and Thurston 03]. Therefore, all arithmetic 3manifolds in the census are known to be large, but there are no general results yet in the nonarithmetic case.

Our requirement for largeness of having a zero Alexander polynomial means that only subgroups with positive first Betti number can be of use in satisfying this condition. Consequently, in order to help with the computations, we restrict attention to the groups in the census having a subgroup with positive first Betti number of index at most 5 . There are 2856 such groups in the census, which is over a quarter of the total, and they can be found quickly using Magma. Our program proves that most of these groups are large; indeed, there are 116 groups left over for which we did not establish largeness within the limited running times. Moreover, there are 132 groups in the census that themselves have positive first Betti number, and a further 305 that have finite first homology but an index-2 subgroup with positive first Betti number. None of these were left over, so we can conclude that a 3-manifold in the closed census with positive first Betti number or that has a double cover with positive first Betti number also has large fundamental group.

\section{DESCRIPTION OF THE ALGORITHM}

Suppose that $G$ is a finitely presented group and $\chi$ is a homomorphism from $G$ onto $\mathbb{Z}$ (which will exist only if the first Betti number $\beta_{1}(G)$ is greater than zero). We define the Alexander polynomial $\Delta_{G, \chi}$ as follows: If $K$ is the kernel of $\chi$, then $G$ acts on $K$ by conjugation. Since the commutator subgroup $K^{\prime}$ of $K$ is characteristic in $G$, we have that $G$ acts by conjugation on the $\mathbb{Z}$-module (i.e., abelian group) $K / K^{\prime}$. But now $K$ acts trivially, so $G / K=\mathbb{Z}$ also acts on $K / K^{\prime}$. This allows us to turn $K / K^{\prime}$ into a $\mathbb{Z}\left[t^{ \pm 1}\right]$-module, where the action of $t$ on an element of $K / K^{\prime}$ is defined to be conjugation by $1 \in \mathbb{Z}=G / K$.

Consequently, if we take a presentation matrix for $K / K^{\prime}$ of $r$ rows (for the relators) and $s$ columns (for the generators), then the Alexander polynomial $\Delta_{G, \chi}(t)$ is defined to be the greatest common factor (up to units $\pm t^{k}$ for $k \in \mathbb{Z}$ ) over all $s \times s$ minors (and if $r<s$, then we add rows of zeros, in which case $\Delta_{G, \chi}$ would be zero). The result is independent of the matrix used to present $K / K^{\prime}$ as a $\mathbb{Z}\left[t^{ \pm 1}\right]$-module.

In order to calculate $\Delta_{G, \chi}$, given a presentation $\left\langle x_{1}, \ldots, x_{n} \mid r_{1}, \ldots, r_{m}\right\rangle$ of $G$ and $\chi$ in the form $\left(a_{1}, \ldots, a_{n}\right) \in \mathbb{Z}^{n}$, where $\chi\left(x_{1}\right)=a_{1}, \ldots, \chi\left(x_{n}\right)=a_{n}$, we apply Fox's free differential calculus (see [Lickorish 97, p. 116]) to form the Alexander matrix $A$, which is an $m \times n$ matrix with entries in $\mathbb{Z}\left[t^{ \pm 1}\right]$. We then calculate all the $(n-1) \times(n-1)$ minors of $A$, namely, the determinants of the submatrices of $A$ formed by deleting one column and the necessary number of rows to make the submatrices square, so it will be $m-n+1$ rows and there will be $n \times\left(\begin{array}{c}m \\ m-n+1\end{array}\right)$ different minors. Then $\Delta_{G, \chi}$ is also the greatest common factor of these minors, and consequently it is 0 if and only if all of the minors are 0 . Again it is independent of the presentation for $G$.

In practice, there is a useful way of reducing by a factor of $n$ the number of minors that have to be calculated. If $I$ is a subset of size $m-n+1$ chosen from $\{1,2, \ldots, m\}$, then let $M_{j}^{I}$ denote the minor with the rows in $I$ removed along with the $j$ th column. We then have the standard identity

$$
M_{j}^{I}\left(1-t^{a_{i}}\right)=M_{i}^{I}\left(1-t^{a_{j}}\right) .
$$

Consequently, if $a_{j}=0$, then $M_{j}^{I}=0$ anyway (by taking $i$ for which $a_{i} \neq 0$ ), and so there is no point in calculating this minor; but otherwise we have $M_{j}^{I}=\delta^{I} \psi_{a_{j}}(t)$, where $\delta^{I}$ is independent of $j$ and $\psi_{k}(t)$ is equal to $\left(1-t^{k}\right) /(1-t)$. This is because only $1-t$ can divide all of $1-t^{a_{1}}, \ldots, 1-t^{a_{n}}$. Consequently, if we take the first column $j$ such that $\chi\left(x_{j}\right) \neq 0$, then we have that $\Delta_{G, \chi}$ is the greatest common factor of the $\delta^{I}$, where $I$ is varied over all possible subsets, thus indicating that we have reduced the number of minors that need to be calculated to $l=\left(\begin{array}{c}m \\ m-n+1\end{array}\right)$.

If we are given a finitely presented group $G$ that we wish to prove is large, then if we can find a finiteindex subgroup $H \leq_{f} G$ and a surjective homomorphism $\chi$ from $H$ to $\mathbb{Z}$ such that the Alexander polynomial $\Delta_{H, \chi}$ with respect to $\chi$ is the zero polynomial, we will be done by [Button 09, Theorem 2.1], which shows that $H$ is large. This result is derived from [Howie 98]; more 
specifically, we have that if $K=\operatorname{ker} \chi$, then the index- $n$ subgroup $K H^{n}$ maps onto $F_{2}$ for all sufficiently large $n$. However, we also have the following result.

Proposition 2.1. If $H$ is a finitely presented group that has a surjective homomorphism $\theta$ to a nonabelian free group $F_{n}$ of rank $n \geq 2$, then we have homomorphisms $\chi$ from $H$ onto $\mathbb{Z}$ with $\Delta_{H, \chi}=0$.

Proof: There are homomorphisms $\chi$ onto $\mathbb{Z}$ that factor through $F_{n}$; take any one of these, so that $\chi=\tilde{\chi} \theta$. Then $\theta$ sends ker $\chi$ onto ker $\tilde{\chi}$, but the free group $F_{n}$ has no nontrivial finitely generated normal subgroups of infinite index, so ker $\tilde{\chi}$ is an infinitely generated free group with $\beta_{1}(\operatorname{ker} \tilde{\chi} ; \mathbb{Q})=\infty$. This implies that $\Delta_{H, \chi}=0$ by [Button 09, Corollary 2.2].

Therefore, this condition of having a finite-index subgroup with zero Alexander polynomial relative to some homomorphism is both necessary and sufficient for a finitely presented group to be large. This enables us now to describe theoretically our partial algorithm.

Recall that there is an algorithm that takes as input a finite presentation and a positive integer $n$ and outputs all the (finitely many) subgroups $H$ having index $n$ in the group $G$ defined by the presentation. This is presented in [Dietze and Schaps 74] and is based on the Todd-Coxeter coset-enumeration process. The output for each $H$ is a list of generators of $H$ and a coset table for the right regular action of $G$ on the cosets of $H$. This allows us by the Reidemeister-Schreier rewriting process to give a finite presentation for $H$.

We now describe in the first part of this section an algorithm that given a finite presentation of a group $G$, works out whether there is a homomorphism $\chi$ such that $\Delta_{G, \chi}=0$. Consequently, it is then clear that we have a partial algorithm for largeness by applying this to each finite-index subgroup of $G$ in turn, terminating with the answer yes as soon as we have found a zero Alexander polynomial. (In fact, practical implementations of the low-index-subgroups routine tend to output subgroups only up to conjugacy, but this is good for us because we are cutting down on duplication.)

We start by calculating the first Betti number $\beta_{1}(G)$, which can be done by abelianizing the given presentation for $G$ and taking the number of $\mathbb{Z}$ summands when $G / G^{\prime}$ is expressed as a finitely generated abelian group. If $\beta_{1}(G)$ is zero, then there are no homomorphisms $\chi$ onto $\mathbb{Z}$, and we must reject $G$ immediately. If $\beta_{1}(G)=1$, then we have just one $\chi$ (up to sign), and the evaluation of
$\Delta_{G, \chi}$ is a straight calculation that involves forming the Alexander matrix above directly from the presentation and then calculating determinants (in fact, we will find that the Alexander polynomial is never zero in this case by Lemma 2.4 , and so we would reject $G$ here too).

However, if $b=\beta_{1}(G) \geq 2$, we have infinitely many $\chi$. Thus we have the advantage of many chances to find a $\chi$ with $\Delta_{G, \chi}$ zero but the disadvantage that we cannot test all of the $\chi$ individually, and so we need a method of narrowing our search.

Theorem 2.2. Given a finitely presented group $G$ with $\beta_{1}(G) \geq 2$, there is an algorithm that determines whether there exists a homomorphism $\chi$ from $G$ onto $\mathbb{Z}$ with $\Delta_{G, \chi}=0$.

Proof: In order to consider all $\chi$ together, we can replace the ring $\mathbb{Z}\left[t^{ \pm 1}\right]$ above with the ring $\mathbb{Z}\left[t_{1}^{ \pm 1}, \ldots, t_{b}^{ \pm 1}\right]$, which is the integral group ring of the free abelianization $\operatorname{ab}(G)=\mathbb{Z}^{b}$ of $G$. We also have a Fox calculus in this case (see [Button 07, Section 2] for an exposition in line with our approach here), and so can form a more general Alexander matrix $B$ with entries in $\mathbb{Z}[\operatorname{ab}(G)]$ and corresponding minors $N_{j}^{I}$.

Since any surjective $\chi: G \rightarrow \mathbb{Z}$ will factor through the natural map $\alpha: G \rightarrow \operatorname{ab}(G)$ and so can be written as $\tilde{\chi} \alpha$, we have that the Alexander matrix $A$ with respect to any given homomorphism $\chi$ is just $B$ evaluated at $\tilde{\chi}$, and consequently the minors $M_{j}^{I}$ are equal to $\left.N_{j}^{I}\right|_{\tilde{\chi}}$.

Thus we calculate these minors $N_{j}^{I}$, which are multivariable polynomials with coefficients in $\mathbb{Z}$, and we now need to consider which $\chi$ will make all the minors vanish. We do this by regarding the minor $N_{j}^{I}$ as a finite subset of lattice points in $\mathbb{Z}^{b}$ with each point weighted by a nonzero integer, where each monomial that appears in $N_{j}^{I}$ with a nonzero coefficient is a lattice point, and the coefficient is the weight. (The ambiguity of units just means that we can shift $N_{j}^{I}$ by unit translations.)

We picture evaluation of $N_{j}^{I}$ at $\tilde{\chi}$ in the following way: we extend $\tilde{\chi}$ to an affine map $\phi: \mathbb{R}^{b} \rightarrow \mathbb{R}$. Then for $x \in \mathbb{R}$ we know that $\phi^{-1}(x)$ is a hyperplane and $N_{j}^{I}$ is zero on evaluation precisely when the following condition is satisfied: for all $m \in \mathbb{Z}$ with $\phi^{-1}(m) \cap N_{j}^{I} \neq \emptyset$, we require that the sum of the weights corresponding to the points of $N_{j}^{I}$ in this hyperplane $\phi^{-1}(m)$ be zero. Let us refer to this situation as " $N_{j}^{I}$ cancels along parallel hyperplanes of constant $\chi . "$

We start with $N_{1}$, which is defined to be the first minor we calculate that happens not to be identically zero (whereas if all minors are identically zero, then we 
conclude that the Alexander polynomial is zero over all homomorphisms). We take any lattice point $\mathbf{x}$ in $N_{1}$. Now $\mathbf{x}$ must cancel with other lattice points in $N_{1}$ on evaluation, so we take every other lattice point $\mathbf{y}$ in turn and join $\mathbf{x}$ and $\mathbf{y}$ by a line $\mathbf{x}+U$, where $U$ is a one-dimensional subspace of $\mathbb{R}^{b}$. We now take the quotient vector space $V=\mathbb{R}^{b} / U$ of dimension $b-1$ and use the quotient map $q$ to regard $N_{1}$ as a finite subset of $V$ with new weights obtained by summing within the translates of $U$.

We now pick a base point of $q\left(N_{1}\right)$, draw a line from it to the other points of $q\left(N_{1}\right)$, and continue recursively. This process stops either because an image of $N_{1}$ is the zero polynomial in dimension $d$, or we reach $d=1$ with a nonzero polynomial. In the latter case, we can reject this $\chi$, but in the former we move on to the next minor $N_{2}$.

We first apply to $N_{2}$ the composition of the quotient maps applied so far, and then we continue in the same way, by picking a base point for this image of $N_{2}$ and another point to which to join it and proceed as before, thus reducing $d$ (or $N_{2}$ might become 0 by dimension $d$, in which case we move on to the next minor and so on).

Eventually, either we have found that all the minors have vanished at dimension $d \geq 1$, in which case any $\chi$ that factors through the composition of quotient maps applied so far will have $\Delta_{G, \chi}=0$, or we reach dimension 1 and conclude that we have failed to reach a suitable $\chi$ along this path, so we must backtrack.

This seems to be an algorithm that could be practical. However, there are areas in which serious improvement can be obtained. Although the above argument should work on minors with few coefficients, the branching nature of this approach means that it will be extremely expensive for large minors (and indeed large numbers of minors). Moreover, we need to calculate all of the minors, but evaluating determinants over multivariable polynomial rings takes considerably longer than in $\mathbb{Z}\left[t^{ \pm 1}\right]$. Furthermore, a big gain comes from looking at the Alexander polynomial modulo a prime $p$ as well as over $\mathbb{Z}\left[t^{ \pm 1}\right]$ (which we refer to as the mod 0 case), as we now describe.

As above, we can let $G / K$ act on the $\mathbb{F}_{p}$ vector space $K / K^{\prime} K^{p}$ and regard this as an $\mathbb{F}_{p}\left[t^{ \pm 1}\right]$ module using the conjugation action of $1 \in G / K=\mathbb{Z}$ as before, and we can also define the $\bmod p$ Alexander polynomial $\Delta_{G, \chi, p}$ as the greatest common factor of the respective minors of any $\mathbb{F}_{p}\left[t^{ \pm 1}\right]$ presentation matrix of the module.

If we want to find $\Delta_{G, \chi, p}$ for a particular $p$, then the new Alexander matrix can be formed merely by reducing modulo $p$ the entries of the original Alexander matrix.
However, if $\bar{\Delta}_{G, \chi}^{p}$ denotes the mod 0 Alexander polynomial $\Delta_{G, \chi}$ with coefficients reduced $\bmod p$, then we have in general that $\bar{\Delta}_{G, \chi}^{p}$ divides $\Delta_{G, \chi, p}$ but not equality (for instance, if we have two determinants $1+t$ and $1+3 t$, then $\Delta_{G, \chi}=1$ but $\Delta_{G, \chi, 2}=1+t$ ).

The advantage of this approach is that the result in [Howie 98] also proves that $G$ is large if there is a prime $p$ with $\Delta_{G, \chi, p}=0$ (in which case we have that the finite-index subgroup $K G^{n}$ maps onto the free product $C_{p} * C_{p} * C_{p}$ for $n$ sufficiently large). Luckily, we have the following:

Proposition 2.3. The reduction $\bar{\Delta}_{G, \chi}^{p}$ of the mod 0 Alexander polynomial is 0 if and only if the mod $p$ Alexander polynomial $\Delta_{G, \chi, p}$ is equal to zero.

Proof: If $\bar{\Delta}_{G, \chi}^{p}=0$, then the only element having zero as a factor is zero, whereas if the greatest common factor in $\mathbb{F}_{p}\left[t^{ \pm 1}\right]$ of all minors is zero, then every element is equal to zero. But the minors in $\mathbb{F}_{p}\left[t^{ \pm 1}\right]$ really are the minors in $\mathbb{Z}\left[t^{ \pm 1}\right]$ reduced mod $p$, so $p$ divides each minor in $\mathbb{Z}\left[t^{ \pm 1}\right]$ and hence divides their common factor, which is $\Delta_{G, \chi}$.

Thus we can still calculate $\Delta_{G, \chi}$, which we regard as a polynomial with integer coefficients, and we have the advantage that largeness is proved if the content of $\Delta_{G, \chi}$ is not equal to 1 .

We now describe the practical approach we adopted for implementation in Magma. Suppose $G$ is the group defined by the input presentation and let $b=\beta_{1}(G)$. We first form the full Alexander matrix $B$ with entries in $\mathbb{Z}\left[t_{1}^{ \pm 1}, \ldots, t_{b}^{ \pm 1}\right]$ using the Fox calculus. This was always quick, even for large presentations and higher values of $b$. We can (and do) tidy up this matrix in multiplying rows by units to clear negative powers. This will change the minors only by units, which means that we can work in the multivariate polynomial ring $\mathbb{Z}\left[t_{1}, \ldots, t_{b}\right]$. We also find the first generator $g$, having infinite image in the abelianization $G / G^{\prime}$ of $G$.

We now split up into cases according to the value of $b$.

Case 1: First Betti number equal to 1. Here we do not in fact calculate $\Delta_{G, \chi}$ for the following reason.

Lemma 2.4. If $G$ is a finitely presented group with $\beta_{1}(G)=1$, and $\chi$ is the unique homomorphism (up to sign) from $G$ onto $\mathbb{Z}$, then $\Delta_{G, \chi}$ is not zero.

Proof: If all of the minors are identically zero, then they continue to be zero when the Alexander matrix $B$ is 
evaluated at $t=1$. However, this is just a presentation matrix for the abelianization $G / G^{\prime}$, which is of the form

$$
C_{d_{1}} \times \cdots \times C_{d_{k}} \times \mathbb{Z} \quad \text { for } d_{1}\left|d_{2}\right| \cdots \mid d_{k} .
$$

Thus the first elementary ideal of $\left.B\right|_{t=1}$, which is an invariant of the abelian group, is $d_{1} \cdots d_{k}$. Since any prime dividing the content of a minor $M$ will also divide $\left.M\right|_{t=1}$, we have that a prime dividing the content of all the minors will divide $d_{k}$.

Consequently, we reject groups whose abelianization is just $\mathbb{Z}$. Otherwise, we obtain the primes $p$ dividing $d_{k}$, and for each one, we work in $\mathbb{F}_{p}[t]$. This is fast because our polynomial ring is a Euclidean domain with a finite coefficient field. Thus we make a copy $A$ of the Alexander matrix $B$ such that the entries of $A$ are coerced into $\mathbb{F}_{p}[t]$, and we cross off the $g$ th column. Since we are in the case of a finitely generated module over a Euclidean domain, we have that $\Delta_{G, \chi, p}=0$ if and only if this smaller matrix has a zero elementary divisor, which is equivalent to its rank being less than the number $n-1$ of columns.

We can use the rank command over this ring to get a quick answer. (Although rank really applies to a matrix over the field of fractions of $\mathbb{F}_{p}[t]$, we can apply the rank command directly here without having to convert.)

This turns out to be a major gain in practice because largeness of a group $G$ can sometimes be achieved using a subgroup $H$ for which $\beta_{1}(H)=1$, for which the calculations will be fast. Indeed, often this will work modulo 2.

Case 2: First Betti number equal to 2. We now describe the algorithm we implemented in MAGMA for this special case. Given a finite presentation of a group $G$ with $\beta_{1}(G)=2$, it is guaranteed to determine in theory whether there exists a homomorphism with a $\bmod p$ or a mod 0 Alexander polynomial that is equal to zero. In practice, we found that it performed quickly too, since it aims to calculate as few determinants over multivariable polynomial rings as possible.

As in the case $\beta_{1}(G)=1$, there is a similar identity between different minors for general $b=\beta_{1}(G) \geq 2$. Let $N_{j}^{I} \in \mathbb{Z}\left[t_{1}^{ \pm 1}, \ldots, t_{b}^{ \pm 1}\right]$ be the minor taken from the multivariable Alexander polynomial $B$ by deleting the rows in $I$ and the $j$ th column, and let

$$
\alpha: \mathbb{Z}[G] \rightarrow \mathbb{Z}\left[t_{1}^{ \pm 1}, \ldots, t_{b}^{ \pm 1}\right]
$$

be the natural extension of the free abelian map from $G$ to $\operatorname{ab}(G)=\mathbb{Z}^{b}$. We have

$$
N_{j}^{I}\left(1-\alpha\left(x_{i}\right)\right)=N_{i}^{I}\left(1-\alpha\left(x_{j}\right)\right)
$$

which implies that $N_{j}^{I}=\delta^{I}\left(1-\alpha\left(x_{j}\right)\right)$, because any common factor of $1-\alpha\left(x_{1}\right), \ldots, 1-\alpha\left(x_{n}\right)$ remains a common factor when evaluated under any homomorphism onto $\mathbb{Z}$.

Consequently, we build the Alexander matrix $B$, and in doing so, we record the vectors $v_{1}, \ldots, v_{n} \in \mathbb{Z}^{2}, v_{j}=$ $\left(a_{j}, b_{j}\right)$, for

$$
\alpha\left(x_{j}\right)=x^{a_{j}} y^{b_{j}} \in \mathbb{Z}\left[x^{ \pm 1}, y^{ \pm 1}\right] .
$$

We first check the two special homomorphisms $\chi_{y}$ and $\chi_{x}$, which means that we set $y$ and then $x$ equal to 1 in $B$ to form the evaluated matrix $B_{y}$ or $B_{x}$. We skip across the columns until we find a generator $x_{j}$ with the first, then second, component of $v_{j}$ being nonzero and delete the $j$ th column (note that if it were zero, then any minor of $B_{y}$ calculated with this column removed would be zero anyway).

We then run through all possible choices of rows that make the resulting matrix square when this column is deleted and look to see whether the determinants vanish. However, we will also want to see whether they all vanish modulo some prime, so we proceed as follows: Let $M^{(1)}=$ $N_{j}^{I_{1}}(x, 1), \ldots, M^{(l)}=N_{j}^{I_{l}}(x, 1)$ be the $l$ minors we have to evaluate, listed in some order. We keep a running value $c$ of the greatest common divisor of the contents of the minors that have been examined so far, which means that all possible primes that might make the minors vanish will be contained in $c$. We do this using the following pseudocode:

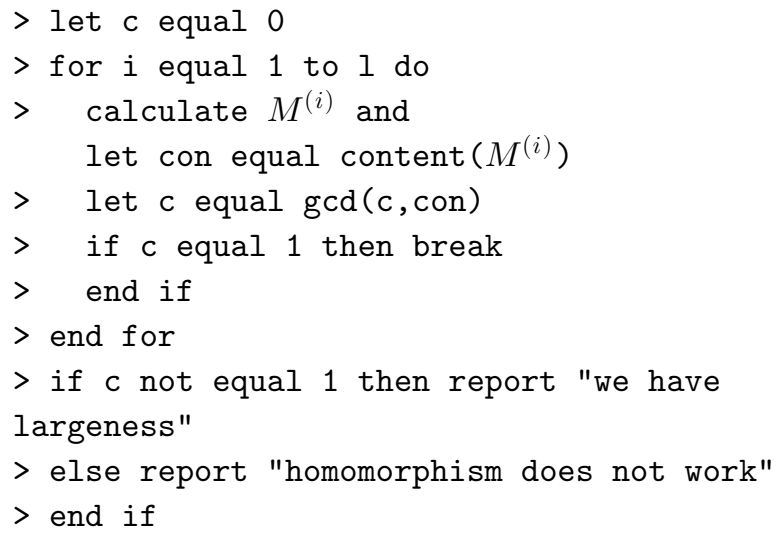

If the homomorphism $\chi_{y}$ fails to establish largeness, then we repeat this process with $\chi_{x}$. At this point we have tried only two homomorphisms, but we can now use a form of the Chinese remainder theorem to test the rest.

Consider the homomorphism $\chi$ sending $(x, y)$ to $\left(t^{l}, t^{m}\right)$, where $\operatorname{gcd}(l, m)=1$. The monomial (or point in $\left.\mathbb{Z}^{2}\right) x^{a} y^{b}$ is sent to $t^{l a+m b}$, so in order for a multivariable 
polynomial $P(x, y)=\sum c_{a, b} x^{a} y^{b}$ to be 0 under evaluation by $\chi$, we need each sum of the $c_{a, b}$ 's over $(a, b)$ such that $l a+m b$ is constant to be zero. If this happens for a particular $\chi$ and a prime $p$ divides $m$ (so does not divide $l$ ), then the set of points $(a, b)$ making up each sum is such that $a$ is constant $\bmod p$. This works for prime powers $p^{i}$, too.

Consequently, we calculate a full minor $N(x, y)$ that is not identically zero. This can be done if we have remembered to record as we went along a list containing the content of each minor evaluated at $\chi_{y}$ that we calculated.

If $M^{(i)}=N_{j}^{I_{i}}(x, 1)$ is the first nonzero minor in this list, then $N(x, y)=N_{j}^{I_{i}}(x, y)$ must be nonzero too, so we calculate this and remove the factor $1-\alpha\left(x_{j}\right)$ to obtain the polynomial $P(x, y)$.

We wish to find out which homomorphisms can make $P$ vanish modulo zero or a prime. Thus for each $p^{i}$ at most the degree of $P(x, y)$ as a polynomial in $x$, we form the "wrapped-up polynomial"

$$
W_{p^{i}}(x, y)=\sum c_{a, b} x^{\bar{a}} y^{b}
$$

where $\bar{a}$ is $a \bmod p^{i}$ (so it is of degree at most $p^{i}-1$ in $x$ ), and we see whether the one-variable polynomial $W_{p^{i}}(x, 1)$ has content equal to 1 .

If so, then we conclude that a homomorphism of the form $(l, m)$ cannot work if $p^{i}$ divides $m$; but if the content is not equal to 1 , then $p^{i}$ is a possible factor of $m$.

We start with $p=2$, then powers thereof, dropping out if a power fails this test and moving on to the next prime. We then record in some set the prime powers that pass as possible factors of $m$, and we should not have many possibilities for $m$. We next do the same for $l$ by swapping $x$ and $y$, then try out all of these $\chi$ to see whether any of them work. This can be done efficiently by evaluating the Alexander matrix under each potential homomorphism so that the entries are in $\mathbb{Z}\left[t^{ \pm 1}\right]$.

We then obtain a column $k$ depending on $\chi$ such that $1-\chi\left(x_{k}\right)$ is not zero in $\mathbb{Z}\left[t^{ \pm 1}\right]$ and remove that column. We can then calculate the minors over all choices of rows to be deleted, and this can be done quickly, since they are single-variable polynomials. We have largeness if the value of $c$ returned by the pseudocode above is not 1 .

However, let $d_{y}$ be the content of $N(x, 1)$, which was the first minor evaluated under $\chi_{y}$ found not to be identically zero. Note that if $d_{y}$ is not equal to 1 , then every prime power $p^{i}$ will pass the above test modulo $d_{y}$ when we wrap up $P(x, y)$, because the homomorphism $\chi_{y}$ is where $(l, m)=(1,0)$, so that all $p^{i}$ will divide $m$. The same is true with $x$ and $y$ swapped, where we set $d_{x}$ to be the content of the first nonzero minor when evaluated under $\chi_{x}$.

We deal with this in the following way: Whenever we are calculating the content of a polynomial that has been obtained by wrapping up $P(x, y)$ above, we remove from the answer any primes that divide $d_{y}$. We do the same for the equivalent polynomial $P^{\prime}(x, y)$ obtained by applying $\chi_{x}$ and $d_{x}$. We then check the candidate homomorphisms $\chi$ obtained from this process to see whether all minors evaluated at $\chi$ are $0 \bmod n$ for some $n$. This is fine, except that it is still possible to have a homomorphism with an Alexander polynomial that is zero modulo a prime $r$, but only if $r$ divides $d_{y}$ or $d_{x}$.

We thus finish by going back to the prime factors $r$ of $d_{y}$ and of $d_{x}$. For each such $r$ we calculate in full two more minors $N^{\prime \prime}(x, y)$ and $N^{\prime \prime \prime}(x, y)$ such that $N^{\prime \prime}(x, 1)$ and $N^{\prime \prime \prime}(1, y)$ both have content coprime to $r$. We will know which minors to choose from our list of contents stored above.

We then run the process above to obtain candidate homomorphisms, but this time we work modulo $r$ throughout. We have finally determined whether there are a homomorphism $\chi$ and a prime $p$ such that all minors evaluated at $\chi$ are $0 \bmod p$.

Case 3: First Betti number equal to 3. Just as moving from $\mathbb{Z}[t]$ to $\mathbb{Z}[x, y]$ means that calculations such as determinants become considerably more expensive in the latter ring, when moving to $\mathbb{Z}[x, y, z]$, we find that things are even worse. Therefore, when $\beta_{1}(G)=3$, our approach was to build the full Alexander matrix $B$ with entries in $\mathbb{Z}[x, y, z]$ and then to evaluate $B$ under the three ring homomorphisms that send $(x, y, z)$ to $(x, y, 1),(x, 1, z)$, and $(1, y, z)$ respectively.

We then fed each of these three double-variable matrices in turn to the $\beta_{1}(G)=2$ routine in Case 2 . This means that the only homomorphisms $(x, y, z) \mapsto$ $\left(t^{l}, t^{m}, t^{n}\right)$ that are being checked are those for which at least one of $l, m, n$ is equal to 0 , and so this process is not guaranteed to find a homomorphism with zero Alexander polynomial if one exists. However, if we regard the space of homomorphisms from $G$ to $\mathbb{Z}$ as $\mathbb{Z}^{\beta_{1}(G)}$, then we can think of Proposition 2.1 as saying that we must have at least a whole 2-dimensional "subspace" of homomorphisms $\chi$ with $\Delta_{G, \chi}=0$ for $G$ to surject onto a nonabelian free group.

Therefore, we will still recognize largeness, because if $\beta_{1}(G)=3$ but $G$ surjects onto $F_{2}$, then there will exist 
a homomorphism of the above special form with zero Alexander polynomial.

Case 4: First Betti number equal to 4 or more. Here we checked only the $b$ canonical homomorphisms of the form

$$
\left(t_{1}, \ldots, t_{b}\right) \mapsto\left(1, \ldots, 1, t_{j}, 1, \ldots, 1\right)
$$

for $j$ between 1 and $b$. This means that we no longer have a routine that is guaranteed to find largeness. We justify this on the grounds that the expense of working with four or more variables is now likely to be prohibitive for all but small presentations. Moreover, the examples we will try out in Sections 3 and 4 have the property that the input group always has first Betti number at most two. It turns out (perhaps fortuitously) that it is very rare for Case 4 to be used, because largeness was nearly always established before reaching such a subgroup.

Finally, we note that when we talk about the algorithm being fast or slow, we are referring throughout to practical rather than theoretical running time. Indeed, if it is the case that the property of largeness is not algorithmically solvable among finitely presented groups (as seems to be the prevailing view), then the theoretical running time for any partial algorithm must be an uncomputable function, since otherwise we could wait until the predicted number of steps to prove largeness had occurred, and then conclude that the input group was not large.

\section{TWO-GENERATOR ONE-RELATOR PRESENTATIONS}

\subsection{Presentations in Magnus Form}

The subclass of finitely presented groups that have a one-relator presentation has been much studied. If such a presentation has at least three generators, then it is well known that the group is large, so it is only twogenerator one-relator presentations that are in doubt. We shall briefly mention the presentations of this form that are known not to yield large groups, but we presented theoretical results in [Button 09] and [Button 10] suggesting that a two-generator one-relator presentation is very often large, and here we will present strong experimental evidence.

Given any two-generator one-relator presentation $\langle x, y \mid w(x, y)\rangle$, we do of course get the same group if we take a conjugate of $w$ or $w^{-1}$. However, there are many other presentations defining the same group, and so to avoid dealing with this, we will consider only presentations in what we call Magnus form. This is based on the fact that there is an automorphism $\alpha$ of $F_{2}$, with let us say $\alpha(x)=a$ and $\alpha(y)=t$, such that $w\left(\alpha^{-1}(a), \alpha^{-1}(t)\right)$ has exponent sum 0 in $t$ when written as a word $w^{\prime}$ in $a$ and $t$. We then say that $w^{\prime}$ is in Magnus form with respect to $t$. This is a consequence of the fact that the kernel of the natural map from $\operatorname{Aut}\left(F_{2}\right)$ to $\operatorname{Aut}\left(F_{2} / F_{2}^{\prime}\right) \cong \mathrm{GL}(2, \mathbb{Z})$ is the group of inner automorphisms of $F_{2}$.

The elementary Nielsen moves on an ordered pair of group elements $\left(g_{1}, g_{2}\right) \in G \times G$ are swapping the pair, replacing either with its inverse, and replacing $g_{1}$ with $g_{1} g_{2}$ or $g_{2}$ with $g_{2} g_{1}$. These moves, when regarded as elements of $\operatorname{Aut}\left(F_{2}\right)$ by their effect on the standard basis $(x, y)$, generate $\operatorname{Aut}\left(F_{2}\right)$. We say that two pairs are Nielsen equivalent if there is a finite sequence of elementary Nielsen moves taking one to the other, so in $F_{2}$ the equivalence class of $(x, y)$ consists precisely of all generating pairs.

If the group $G$ is given by a presentation $\langle a, t \mid w(a, t)\rangle$ with $w$ cyclically reduced and in Magnus form with respect to $t$, then we can keep $w$ in this form by replacing $t$ with $t a^{k}$ for any $k \in \mathbb{Z}$ (or sending $t$ or $a$ to its inverse), but $\operatorname{Out}\left(F_{2}\right)$ being isomorphic to $\mathrm{GL}(2, \mathbb{Z})$ implies that these are the only automorphisms of $F_{2}$ that we can make up to conjugation that preserve Magnus form (at least if $\beta_{1}(G)=1$ : if $\beta_{1}(G)=2$, then every two-generator onerelator presentation of $G$ is in Magnus form with respect to both generators). However, as we shall see later, there exist two-generator one-relator groups with more than one Nielsen equivalence class of generating pairs.

Therefore, given any two-generator one-relator presentation, we can assume that $w$ is of the form

$$
t^{k_{1}} a^{l_{1}} \cdots t^{k_{n}} a^{l_{n}},
$$

where $k_{i}, l_{i} \neq 0$, and $k_{1}+\cdots+k_{n}=0$ (excluding the words $a^{l}$ ). Thus writing $a_{i}=t^{i} a t^{-i}$, we have

$w=a_{s_{1}}^{l_{1}} a_{s_{2}}^{l_{2}} \cdots a_{s_{n}}^{l_{n}}$, where $s_{1}=k_{1}$ and $s_{i+1}=s_{i}+k_{i+1}$.

We call $h=\max \left(s_{i}\right)-\min \left(s_{i}\right)$ the height of $w$ when it is in Magnus form with respect to $t$, and $2 n$ the syllable length of $w$. Note that the moves above preserving Magnus form also preserve the height and the syllable length, though not necessarily the word length, of $w$.

The presentations that are known not to be large all fall into two types: First, if the syllable length is 4 with $k_{1}=-k_{2}=1$, then we have the Baumslag-Solitar group $\operatorname{BS}\left(l_{1},-l_{2}\right)$, and it is well known that this is not large if and only if $l_{1}$ and $l_{2}$ are not coprime. The other type comes from [Button 10, Theorem 4.3] (based originally on a construction in [Higman 51]; see also [Baumslag et al. 07]), which states that if $g$ and $h$ 
are conjugate elements of a group $G$ where the relation $h^{k} g^{l} h^{-k}=g^{l \pm 1}$ holds in all finite images of $G$, then $g$ and $h$ must be trivial in any finite image of $G$. In particular, if $G=\left\langle a, t \mid v^{k} a^{l} v^{-k}=a^{l \pm 1}\right\rangle$, where $v$ is an element of $F_{2}$ that is conjugate to $a$, then the presentation is in Magnus form with respect to $t$, but all finite images are cyclic, so $G$ is not large.

Taking $k=l=1$ and the plus sign with $v=$ $t a t^{-1}$, we obtain the famous group first introduced in [Baumslag 69]. Note that further examples can be obtained by iterating this construction because if $G=$ $\langle a, t \mid w\rangle$ is a presentation in which $a$ is trivial in every finite image of $G$, then we can take $w$ and a conjugate of $w$ to form a new relator in which $w$ is trivial in every finite image of this new group, and thus $a$ is too.

We also remark that these nonlarge groups (as well as some others) typically have unusual presentations that are not Nielsen equivalent to the well-known ones. This observation dates back to [McCool and Pietrowski 71] and is based on the following trick: if $G=\langle x, y \mid w(x, y)\rangle$ is such that we can write $w$ in the form $u(x, y)^{k}=$ $y$, then by introducing the letter $z=u(x, y)$, we have that $G$ is also $\left\langle x, z \mid z=u\left(x, z^{k}\right)\right\rangle$, which for $k \neq 0, \pm 1$ is generally not Nielsen equivalent. For instance, taking the presentation $\left\langle x, y \mid\left(x y^{r} x^{-1} y^{ \pm r}\right)^{k}=y\right\rangle$, we get $\left\langle x, z \mid x z^{k r} x^{-1} z^{ \pm k r}=z\right\rangle$, so we have an alternative presentation for $\mathrm{BS}(k r, \mp k r+1)$. These are in Magnus form with respect to $x$ but have longer syllable length than the usual presentation, so cannot be Nielsen equivalent. Also, it was shown in [Brunner 76] that for any $s \geq 1$, the groups

$$
\left\langle a, t \mid\left(t a^{2^{s}} t^{-1}\right) a\left(t a^{2^{s}} t^{-1}\right)^{-1}=a^{2}\right\rangle
$$

are all isomorphic to Baumslag's example by putting $b=$ $a^{2}$, so that $a=\left(t b^{2^{s-1}} t^{-1}\right)^{-1} b\left(t b^{2^{s-1}} t^{-1}\right)$, thus giving the same relation with $b$ and $s-1$ in place of $a$ and $s$.

This is also one of a family of examples in [Brunner 80]:

$$
D(k, l, m)=\left\langle a, t \mid\left(t a^{k} t^{-1}\right) a^{l}\left(t a^{k} t^{-1}\right)^{-1}=a^{m}\right\rangle,
$$

so that Baumslag's example is $D(1,1,2) \cong D\left(2^{s}, 1,2\right)$ for $s \geq 0$. Note that $D(k, l, m)$ is large by the Alexander polynomial if $|l-m| \neq 1$ and has only finite cyclic quotients otherwise by the above. Also, for the sake of Table 1 , we point out that not only is $D(k, l, m)$ isomorphic to $D(-k,-l,-m)$ by sending $a$ to $a^{-1}$, but also to $D(-k, l, m)$ by further inverting both sides of the relation. Therefore we can assume that $k$ and $l$ are always positive.

\subsection{Height-1 Presentations}

Let us suppose that the height of a presentation in the form $(3-1)$ is 1 , so that $n$ is even and (by sending $t$ to $t^{-1}$ if necessary) $k_{i}$ is 1 for $i$ odd and is -1 for $i$ even. Here we do not need anything as involved as in Section 2 to determine largeness because [Button 10, Corollary 4.2] tells us that $G$ is large if and only if there is $H \leq_{f}$ $G$ with $d\left(H / H^{\prime}\right) \geq 3$, where $d$ is the minimum number of generators for a group. Therefore we merely need to compute for each $i \geq 2$ the index- $i$ subgroups of $G$ and their respective abelianizations, breaking off as soon as one is found needing at least three generators.

When we have done this for reasonably high $i$, we can look at which presentations are left and ask whether we already recognize them as groups known not to be large. To do this efficiently in such a way that we are not repeatedly taking the same group with many different presentations but without obsessively demanding only one presentation per group, we adopt the following technique: First note that for height-1 presentations the move $t \mapsto t a^{k}$ does not change $(3-1)$. Then by taking cyclic permutations and sending $a$ to $a^{-1}$ if necessary, we can assume that $l_{1}>0$ and $l_{1} \geq\left|l_{i}\right|$ for all $i$. We can further arrange it by reversing the word that $l_{2}>l_{n}$, or if equal that $l_{3}>l_{n-1}$, and so on. We then choose an upper bound for the total word length that we will consider, which in our case was 14 . Then for syllable length $2 n$ (starting at $n=4$ because $n=2$ yields only Baumslag-Solitar groups) and a fixed $l_{1}$ (starting at 1 ), we have an upper bound $b$ on the moduli of the other $l_{i}$ (so that $b$ will equal $l_{1}$, or less if that value always makes the word too long).

Thus we take all presentations for which the values of $\left(l_{2}, \ldots, l_{n}\right)$ are counted (ignoring zeros) from $(-b, \ldots,-b)$ to $(b, \ldots, b)$, and we input them if the word length is at most 14 . We then choose a bound for the index of the subgroups we examine that is as high as possible without long delays in finding all subgroups of this index: since some groups will be proved large well before this, we went up to index 12 , which with these presentations usually took only a few seconds.

This allows us to draw up an initial list of what might fail to be large. The policy adopted from then on was as follows: First find the Alexander polynomials and try to show that the group is $\operatorname{BS}(m, n)$ or $D(k, l, m)$ by use of the transformations above. If this fails, then it is also a consequence of [Button 10, Section 4] that if $G$ is given by a height- 1 presentation but is not large, then the finite residual $R_{G}$ is equal to $G^{\prime \prime}$. If $G$ has the same Alexander polynomial as $B=\operatorname{BS}(m, n)$, then since $R_{B}=B^{\prime \prime}$ too with $G / G^{\prime \prime}$ isomorphic to $B / B^{\prime \prime}$, any finite-index 
subgroup of $B / B^{\prime \prime}$ is also one of $G / G^{\prime \prime}$ and hence corresponds under the inverse image to a unique finite-index subgroup of $G$ and of $B$ too. Moreover, these subgroups will have the same abelianization throughout, since if $H \leq_{f} G$, then

$$
H^{\prime \prime} \geq R_{H}=R_{G}=G^{\prime \prime} \geq H^{\prime \prime},
$$

so $H^{\prime \prime}=G^{\prime \prime}$, meaning that the abelianization of the corresponding subgroup $H / G^{\prime \prime}$ of $G / G^{\prime \prime}$ is

$$
\left(H / G^{\prime \prime}\right) /\left(H^{\prime} G^{\prime \prime} / G^{\prime \prime}\right) \cong H / H^{\prime} G^{\prime \prime}=H / H^{\prime},
$$

which is equal to that of $H$. Also the same holds for finite-index subgroups of $B$, so we compare the abelianizations of subgroups of $G$ and of $B$ up to index 12, and even though it may be that all such subgroups $H$ of $G$ have $d\left(H / H^{\prime}\right)=2$, the torsion of $H / H^{\prime}$ might have higher order than the torsion of the abelianization of the equivalent subgroup of $B$. This worked for most of the remaining presentations, with for some reason index-7 subgroups very often successful.

The upshot was that this process left only four presentations unknown as to whether they were large.

Table 1 lists all height-1 presentations of length up to 14 giving rise to nonlarge groups, in order of syllable length (starting at 4) and then word length, along with these four.

We could resolve two of these presentations: they were (using capital letters for inverses)

$$
G_{1}=\left\langle a, t \mid t a^{3} T a^{2} t a^{3} T A\right\rangle
$$

and

$$
G_{2}=\left\langle a, t \mid t a^{3} \operatorname{Tata}^{3} T A^{2}\right\rangle .
$$

For height-1 presentations it is enough to find a single finite image that is (nonabelian) simple, since then we do not have $R_{G_{i}}=G_{i}^{\prime \prime}$, so $G_{1}$ and $G_{2}$ are large by [Button 10, Theorem 4.1]. The computer found that $G_{1}$ maps onto the unitary group $\mathrm{U}(3,3)$ of order 6048 , and $G_{2}$ has the sporadic simple group $J_{2}$ of order 604800 as a finite image.

However, that still leaves two groups whose Alexander polynomial is 1 , so largeness is determined by the answer to the following question:

Question 3.1. For the groups with presentations

$$
\left.\langle a, t| t a^{2} \text { TatATatATA }\right\rangle
$$

and

$$
\left\langle a, t \mid t a^{2} T a t A T A t A T A\right\rangle,
$$

is it true that $a$ is trivial in every finite image?
Consequently we have established that the vast majority of two-generator one-relator height-1 presentations with short word length define large groups. However, the only way we determined that some groups are not large was to show them to be isomorphic to groups already known to have this property, and there seems to be a very limited number of methods that are able to prove that presentations of this type are not large.

\subsection{General Height Presentations}

We now consider two-generator one-relator presentations $\langle a, t \mid w(a, t)\rangle$ in Magnus form with respect to $t$ but with height greater than 1 . Here we need to implement using Magma the algorithm described in Section 2. Before we embark on running it, we note that not all presentations of this type define large groups. The only example we know of already in the literature is

$$
\left\langle a, t \mid\left(t^{k} a t^{-k}\right) a\left(t^{k} a t^{-k}\right)^{-1}=a^{2}\right\rangle
$$

in [Moldavanskiur and Sibyakova 95], which has height $k$.

However, since $t^{k}$ can be replaced here by any word in $F_{2}$, it is clear that there are many more such presentations. Given that the above example provides one of length 13 , we look in this subsection at presentations of word length at most 12 , and it seems surprising that we will not encounter any nonlarge examples.

We had to work much harder than in the height-1 case. However, an important point that makes these presentations tractable is that although a finite-index subgroup of a two-generator one-relator group will not usually have such a presentation itself, it will still have a deficiency-1 presentation, so that for each subgroup only one minor need be calculated.

For a given $n$ we fixed a $t$-shape, which we define to be the vector $\left(k_{1}, \ldots, k_{n}\right)$ in the expression $(3-1)$, and we had a bound $b$ such that if any exponent $l_{i}$ of the letter $a$ had modulus greater than $b$, then the word was too long. We then input all $a$-shapes $\left(l_{1}, \ldots, l_{n}\right)$ from $(-b, \ldots,-b)$ to $(b, \ldots, b)$ if the resulting word length was at most 12 . Note that because we can send $a$ to $a^{-1}$ in a presentation, each group has been input at least twice. This is no bad thing, because it acts as a check, and it could happen that one presentation was proved large, whereas the other was missed, although this anomaly can come about only on subgroups with first Betti number at least 3, as described in Section 2.

The initial results, when run up to index 12 , were that there was one remaining presentation not proved large of length 9, four of length 10, fewer than thirty of length 11 , and none of length 12 . We then ran the program on 
these remaining presentations on subgroups of index 13, 14 , and 15 . It would take about a minute to obtain all the subgroups of index 15, and the Alexander polynomial checks would then be instant, although the time taken to rewrite for a subgroup presentation was occasionally significant.

We were then left with only one length-9 presentation and fifteen length-11 presentations, although some of these were seen to be isomorphic to each other. We then needed to find other ways of showing that these groups are large.

One option is use the MAGMA command LowIndexNormalSubgroups, which will be able to find normal subgroups up to a higher index than that for arbitrary subgroups, but there will be fewer of them. The main tool we use is a form of bootstrapping: Pick a low-index subgroup $H$ with decent abelianization, by which we usually mean $\beta_{1}(H)$ at least 2 or (though preferably and $d\left(H / H^{\prime}\right) \geq 3$. Then rewrite to get a finite presentation for $H$ and regard this as our input. Although the presentation will get longer, so that we will be unable to find all subgroups up to index 15 again, a subgroup $L$ of $H$ will have index $[G: H][H: L]$ in $G$, so we can go considerably higher by being selective. We can even repeat the process until the calculations become too long.

Let us discuss the solitary presentation of length 9 that was left over, sice this turns out to be an interesting group that has already appeared in the literature. The presentation is $\left\langle a, t \mid t^{3} a t^{-2} a^{-1} t^{-1} a^{-1}\right\rangle$ and the group $\Gamma$ it defines is free-by-cyclic, since its homomorphism onto $\mathbb{Z}$ has kernel the free group $F_{3}$ of rank 3 . This can be seen by setting $b=t a t^{-1}$ and $c=$ $t b t^{-1}$, so that we have an alternative presentation of the form

$$
\Gamma=\left\langle a, b, c, t \mid t a t^{-1}=b, t b t^{-1}=c, t c t^{-1}=a b\right\rangle,
$$

which happily is the subject of the paper [Stallings 82], in which it is shown that the corresponding automorphism $\phi$ of $F_{3}$ is not topologically realizable as a homeomorphism of a compact surface with boundary. This was followed up in the paper [Gersten and Stallings 91], in which conditions were given to ensure that all positive powers of an automorphism $\alpha$ of the free group $F_{n}$ are irreducible, where $\alpha$ is said to be reducible if there exist proper nontrivial free factors $R_{1}, \ldots, R_{k}$ of $F_{n}$ such that the conjugacy classes of $R_{1}, \ldots, R_{k}$ are permuted transitively by $\alpha$ (see [Bestvina and Handel 92]). In particular, it was shown that all positive powers of $\phi$ are irreducible, and furthermore that for $k \geq 1$, no power $\phi^{k}$ can fix a nontrivial word, even up to conjugacy.

As for largeness of groups $G=F_{n} \rtimes_{\alpha} \mathbb{Z}$ for $n \geq 2$, this is true by [Button 09] if $G$ contains $\mathbb{Z} \times \mathbb{Z}$, which is equivalent to there being a nontrivial element $w$ in $F_{n}$ and $k \geq 1$ such that $w$ is sent by $\alpha^{k}$ to a conjugate of itself. It is also equivalent to a group of this form not being word-hyperbolic.

Thus our group $\Gamma$ above is word-hyperbolic. However, we can have cases in which $G$ is not word-hyperbolic but all powers of $\alpha$ are irreducible; for example, if $\alpha$ is an automorphism of $F_{2}=\langle x, y\rangle$, then $\alpha^{2}$ will always fix the conjugacy class of $x y x^{-1} y^{-1}$, but $\alpha^{k}$ need not fix a generator of $F_{2}$ for any $k$. We can also have $G$ being word-hyperbolic but $\alpha$ reducible; indeed, in [Button 10] the first example of a large word-hyperbolic group of the form $F_{n} \rtimes_{\alpha} \mathbb{Z}$ is given by putting together two copies of Stallings's automorphism $\phi$. However, since the computer managed to show that $G$ is large (by finding a subgroup $H$ of index 56 , where $\beta_{1}(H)=4$, and a homomorphism $\chi$ with $\Delta_{H, \chi}=0 \bmod 2$ ), we have the first large example in the following theorem:

Theorem 3.2. The word-hyperbolic group $\Gamma=F_{3} \rtimes_{\phi} \mathbb{Z}$ defined above is large, even though $\phi$ is irreducible with irreducible powers.

Details of the arguments that established largeness for these 16 remaining presentations can be found in [Button 08]. From our computations we have established the following result:

Theorem 3.3. If $G$ is given by a two-generator one-relator presentation $\langle x, y \mid r\rangle$ in Magnus form and $r$ is cyclically reduced with length at most 12 , then $G$ is not large if and only if either the presentation appears in Table 1 (up to cyclic permutation of $r$ or $r^{-1}$ and replacing either generator by its inverse), or the presentation is of the form $\left\langle a, t \mid t a^{p} t^{-1}=a^{q}\right\rangle$, where $p$ and $q$ are coprime.

This also gives the full picture for the virtual first Betti number of these groups:

Corollary 3.4. If $G$ is as in Theorem 3.3, then $G$ has infinite virtual first Betti number unless the presentation appears in Table 1 (up to cyclic permutation of $r$ or $r^{-1}$ and replacing either generator by its inverse), in which case the virtual first Betti number is 1 ; or the presentation is of the form $\left\langle a, t \mid t a^{p} t^{-1}=a^{q}\right\rangle$, where $p$ and $q$ are 
coprime, in which case the virtual first Betti number is 2 for $p, q= \pm 1$ and 1 otherwise.

Corollary 3.5. If $G$ is as in Theorem 3.3 with $r$ having length at most 12 and height at least 2 , then $G$ is large.

As noted at the start of this subsection, this is not true once we move to length 13 . However, note that all groups that have been shown not to be large contain Baumslag-Solitar subgroups and so cannot be (nonelementary) word-hyperbolic groups.

\subsection{First Betti Number Equal to 2}

It may have been noticed in this section that if the relator $r$ is in the commutator subgroup $F_{2}^{\prime}$ (which is equivalent to the group $G=\langle x, y \mid r\rangle$ having $\beta_{1}(G)=2$ ), then no examples have yet been found in which $G$ is not large, except for $r=[x, y]=x y x^{-1} y^{-1}$. Although it is possible for $G$ not to be large and not equal to $\mathbb{Z} \times \mathbb{Z}$ in this case, such as the example

$$
\left\langle x, y \mid\left[y^{-1}, x\right][x, y]\left[y^{-1}, x\right]^{-1}=[x, y]^{2}\right\rangle
$$

in [Baumslag et al. 07, Corollary 2], there is theoretical evidence that $G$ is often large; the strongest result in this area is [Button 09, Theorem 3.6], stating that if $r$ is actually a commutator and there is $H \leq_{f} G$ with $d\left(H / H^{\prime}\right) \geq 3$, then $G$ is large (and if the condition on $H$ is removed, then the only known counterexample is $\mathbb{Z} \times \mathbb{Z}$ ).

In this subsection we describe our work that shows that if $G=\langle x, y \mid r\rangle$ for $r \in\left(F_{2}\right)^{\prime}$ with word length at most 16 , then either $G=\mathbb{Z} \times \mathbb{Z}$ (which is well known to happen only if $r=[x, y]$ when reduced and cyclically reduced, up to a cyclic permutation of $r$ or of $r^{-1}$, so $r$ always has length 4 ) or $G$ is large. The example given above suggests that this would not be true for word length 20; in fact, writing this word out reveals that it has length 18 . While it might not be remarkable that no counterexamples exist that have length shorter than 18, we also managed to show with calculations on a substantial number of cases the somewhat more surprising result that this is the only counterexample among words of length 18 .

Given $r \in F_{2}^{\prime}$, it must have even length. From Theorem 3.3 we are fine for length at most 12 , and in fact for length 14, too: on running the program again applied to relators of length 14 but subject to the condition that the exponent sum of $a$ is zero, we very quickly find that all such presentations give large groups.

Moreover, we have by the combined results of [Edjvet 84] and [Button 09] that $G$ is always large (or $\mathbb{Z} \times \mathbb{Z})$ if $r \in F_{2}^{\prime}$ and has syllable length 4 or 6 . There- fore when we move on to length 16 we need only consider words with eight syllables or more. We have that $r$ is automatically in Magnus form with respect to both $a$ and $t$, and remains so under any Nielsen transformation, although the word length can change. We therefore assume that $k_{1}>0$ and that $k_{1}$ has largest modulus among both the $k_{i}$ and the $l_{i}$ (otherwise, we could swap $a$ and $t$ ). We then fix the $k_{i}$ while counting through the various $l_{i}$ as in Theorem 3.3 but subject to $l_{1}+\cdots+l_{n}=0$ and word length exactly 16.

Here we begin to encounter the problem that rewriting can take a long time. There is also a tiny number of presentations that complete the rewriting process on all subgroups up to index 12 without being proved large. We then use ad hoc arguments on the remainder as above, but the same ideas will work on these too. Once again the details are in [Button 08].

We then move up to length 18 and proceed in the same way. Here the main consumption of time is taken up by inputting each $t$-shape from the long list of possibilities and waiting for the program to finish (or occasionally get stuck, whereupon we would try a different strategy).

We find that all cases are proved large apart from those in which the $t$-shape is $(2,-1,-1,2,-1,1,-1,-1)$ and the exponents of $a$ have modulus 1 and alternate in sign, which is our one exception given earlier. Thus we have a result that is definitive for words in the commutator subgroup with length at most 18 .

Theorem 3.6. Suppose $G$ is given by a two-generator onerelator presentation $\langle x, y \mid r\rangle$ with $r$ cyclically reduced and in the commutator subgroup of $F_{2}$, with the word length of $r$ at most 18 . Then $r$ has word length 4 and $G$ is isomorphic to $\mathbb{Z} \times \mathbb{Z} ; r$ has word length greater than 4 but less than 18, in which case $G$ is large; or $r$ has word length 18 and $G$ is large except for

$$
r=\left[y^{-1}, x\right][x, y]\left[y^{-1}, x\right]^{-1}[x, y]^{-2},
$$

up to cyclic permutation of $r$ or $r^{-1}$ and replacing either generator by its inverse, whereupon $G$ contains a nonabelian free group but all finite images of $G$ are abelian and all finite-index subgroups of $G$ have abelianization $\mathbb{Z} \times \mathbb{Z}$.

\section{CLOSED HYPERBOLIC 3-MANIFOLDS}

The other examples on which we tried out our program were the fundamental groups of closed orientable hyperbolic 3-manifolds (hereinafter, we refer to these as 3-manifold groups), and again we met with some 
success. It is an open question whether every 3-manifold group is large. The focus of our work was the closed census of hyperbolic 3-manifolds [Hodgson and Weeks 01] that accompanies the program SnapPea. This is available as a database in MAGMA of 11,126 entries, each of which contains a presentation of the corresponding 3-manifold group, along with the manifold's name and volume (which provides an ordering for the database). A 3-manifold group always has a presentation of deficiency 0 (and never has a presentation of strictly positive deficiency). In the census we find that most presentations are of two-generator two-relator type with a fair number of three-generator three-relator presentations, mainly concentrated among the higher-volume manifolds.

Consequently, we have no guarantee that the group has a finite-index subgroup with positive first Betti number. Since our program merely ignores finite-index subgroups that have no homomorphisms onto $\mathbb{Z}$, there would be no point in entering a group if no such subgroup were already known or easily found.

Fortunately, this has already been covered in the paper [Dunfield and Thurston 03], in which it was proved that all 3-manifold groups in this census have positive virtual first Betti number. However, this was a major computational undertaking involving about a year of CPU time. The final group to be completed was found to have this property by running MAGMA to obtain its subgroups of index 14 and then examining the abelianizations. This took two days, which was about the time we had available to look at the whole census. Moreover, the highest index of a subgroup that provided positive virtual first Betti number of a group was 515,100 , which (although this may not be the minimal index for this group) is far too high to expect the computer to provide a presentation to check for largeness. The paper [Dunfield and Thurston 03] exploited the fact that one wants to know only whether the abelianization of a subgroup is infinite, rather than needing explicitly to find the rank and torsion, and there some representation theory of finite groups was utilized.

Therefore, the approach we adopted was to look for largeness among groups that have a very low index subgroup with infinite abelianization. Trying this out with index at most 5, we found in less than two minutes that there are 2856 groups with this property, which at just over a quarter is a decent proportion. The choice of 5 reflected our desire for a bound that ensured that we were covering a large enough sample of the census in our program, but was also chosen because we ran our largeness routine up to index 10 on all of these groups. The reason for going up to double the initial choice of index is that because a group with a homomorphism onto $\mathbb{Z}$ has all finite index subgroups with the same property, we knew that we would have more than one subgroup of the original group with positive Betti number that could be checked for largeness. (Note that the proportion of groups having a low-index subgroup with positive first Betti number is higher here than in [Dunfield and Thurston 03], because there, other methods were first used to find such subgroups, rather than the enumeration of all low-index subgroups.)

We then ran the routine described in Section 2 on this list of 2856 3-manifold groups, checking subgroups up to index 10. The results were encouraging. We ran the program in batches of a few hundred at a time, and only 130 failed to be proved large by this method. Some 3manifold groups were dealt with very quickly, although several toward the end took quite a long time (there were three cases in which we gave up on finding the index-10 subgroups after waiting for over an hour).

For a few of the very early 3-manifold groups with more tractable presentations, we increased the index for finding subgroups up to 12 or 14 . This produced four other large groups in our sample. We then used the data in Snap ${ }^{1}$ to see whether any 3 -manifold groups in the leftover list were arithmetic. We did so because every arithmetic 3-manifold group in the census is large, which can be seen by combining [Lackenby et al. 08 , Theorem 6.1] (an arithmetic 3-manifold group with a finiteindex subgroup having first Betti number at least 4 is large), [Cooper et al. 07] or [Venkataramana 08] (an arithmetic 3-manifold group with positive virtual first Betti number has infinite virtual first Betti number), and [Dunfield and Thurston 03] (every census 3-manifold has positive virtual first Betti number). This provided eight more examples, although they all came from early in the census. Combining the data, we were left with 118 3manifold groups left over that were not proved large.

Among these 118 were two groups that themselves have positive first Betti number, out of 132 in the database. We pushed up the index to 11 or 12 here and established largeness for this pair. Moreover, we observe that all of the 305 further 3-manifolds in the census having a double cover with positive first Betti number have been proved large, giving the following theorem.

Theorem 4.1. If $G$ is the fundamental group of a closed hyperbolic 3-manifold in the census and $\beta_{1}(G) \geq 1$ or $G$

\footnotetext{
${ }^{1}$ Available at http://www.ms.unimelb.edu.au/ $\sim_{\text {snap } / .}$
} 
has a double cover with positive first Betti number, then $G$ is large.

Details are given in [Button 08] on how to determine whether a given census 3-manifold is one of the 2740 that have here been proved large.

One point to mention: a finite-index subgroup of a 3-manifold group always has a presentation of deficiency zero, because it is itself a 3-manifold group, being the fundamental group of a finite cover of a 3-manifold. However, the computer does not know this, and often on rewriting we obtained presentations of strictly negative deficiency ( -4 was the lowest value observed, with -1 appearing quite often and -2 or -3 cropping up occasionally). The approach we first adopted during the runs was to ignore these subgroups and extend our routine for deficiency-1 group presentations just to the case of deficiency 0 , because we feared the running time might otherwise be substantially lengthened owing to the larger number of minors that need to be calculated. However, this was overcautious, because when the program was extended to the version described in Section 2 that allows presentations of arbitrary deficiency, it then ran more quickly, because lower-index subgroups could establish largeness earlier.

Update: After the first version of this paper, we further developed the routine during a visit to the MAGMA research group. In particular, the bootstrapping described in Section 3.3 was incorporated automatically, and this significantly improved the performance. An attack on the first 5003 -manifolds in the census using the newer code managed to establish largeness for all of these apart from three cases: numbers 75,268 , and 304 . We hope that this routine will be available in a future release of MAGMA.

\section{REFERENCES}

[Baumslag 69] G. Baumslag, "A Non-cyclic One-Relator Group All of Whose Finite Quotients Are Cyclic." J. Austral. Math. Soc. 10 (1969), 497-498.

[Baumslag et al. 07] G. Baumslag, Charles F. Miller III, and D. Troeger. "Reflections on the Residual Finiteness of One-Relator Groups." Groups Geom. Dyn. 1 (2007), 209219.

[Bestvina and Handel 92] M. Bestvina and M. Handel. "Train Tracks and Automorphisms of Free Groups." Ann. of Math. 135 (1992), 1-51.

[Brunner 76] A. M. Brunner. "A Group with an Infinite Number of Nielsen Inequivalent One-Relator Presentations." J. Algebra 42 (1976), 81-84.
[Brunner 80] A. M. Brunner. "On a Class of One-Relator Groups." Canad. J. Math. 32 (1980), 414-420.

[Button 07] J. O. Button. "Mapping Tori with First Betti Number at Least Two." J. Math. Soc. Japan 59 (2007), 351-370.

[Button 09] J. O. Button. "Large Groups of Deficiency 1." Israel J. Math. 167 (2009), 111-140.

[Button 10] J. O. Button. "Largeness of LERF and 1-Relator Groups." Groups Geom. Dyn. (2010), 709-738.

[Button 08] J. O. Button. "Proving Finitely Presented Groups Are Large by Computer" (arxiv version). arxiv.org/abs/math/0812.4264, 2008.

[Cooper et al. 07] D. Cooper, D. D. Long, and A. W. Reid. "On the Virtual Betti Numbers of Arithmetic Hyperbolic 3-Manifolds." Geom. Topol. 11 (2007), 2265-2276.

[Dietze and Schaps 74] A. Dietze and M. Schaps. "Determining Subgroups of a Given Index in a Finitely Presented Group." Canadian J. Math. 26 (1974), 769-782.

[Dunfield and Thurston 03] N. M. Dunfield and W. P. Thurston. "The Virtual Haken Conjecture: Experiments and Examples." Geom. Topol. 7 (2003), 399-441.

[Edjvet 84] M. Edjvet. "The Concept of 'Largeness' in Group Theory." PhD thesis, University of Glasgow, 1984.

[Gersten and Stallings 91] S. M. Gersten and J. R. Stallings. "Irreducible Outer Automorphisms of a Free Group." Proc. Amer. Math. Soc. 111 (1991), 309-314.

[Higman 51] G. Higman. "A Finitely Generated Infinite Simple Group." J. London Math. Soc. 26 (1951), 61-64.

[Hodgson and Weeks 01] C. D. Hodgson and J. R. Weeks. "Closed Census Invariants." Available online (ftp:// www.geometrygames.org/priv/weeks/SnapPea/SnapPea Census/ClosedCensus/ClosedCensusInvariants.txt), 2001.

[Holt and Rees 96] D. F. Holt and S. Rees. "Free Quotients of Finitely Presented Groups." Experiment. Math. 5 (1996), $49-56$.

[Howie 98] J. Howie. "Free Subgroups in Groups of Small Deficiency." J. Group Theory 1 (1998), 95-112.

[Lackenby et al. 08] M. Lackenby, D. D. Long, and A. W. Reid. Covering Spaces of Arithmetic 3-Orbifolds, Int. Math. Res. Not. IMRN 12. Cary, NC: Oxford University Press, 2008.

[Lickorish 97] W. B. R. Lickorish. An Introduction to Knot Theory, Graduate Texts in Mathematics 175. New York: Springer, 1997. 
[Lyndon and Schupp 77] R. C. Lyndon and P. E. Schupp. Combinatorial Group Theory. New York: Springer, 1977.

[McCool and Pietrowski 71] J. McCool and A. Pietrowski. "On Free Products with Amalgamation of Two Infinite Cyclic Groups." J. Algebra 18 (1971), 377383 .

[Moldavanskiŭ and Sibyakova 95] D. Moldavanskiu and N. Sibyakova. "On the Finite Images of Some One-Relator
Groups." Proc. Amer. Math. Soc. 123 (1995), 20172020 .

[Stallings 82] J. R. Stallings. "Topologically Unrealizable Automorphisms of Free Groups." Proc. Amer. Math. Soc. 84 (1982), 21-24.

[Venkataramana 08] T. N. Venkataramana. "Virtual Betti Numbers of Compact Locally Symmetric Spaces." Israel J. Math. 166 (2008), 235-238.

J. O. Button, Selwyn College, University of Cambridge, Cambridge CB3 9DQ, U.K. (jb128@dpmms.cam.ac.uk)

Received December 22, 2009; accepted in revised form November 24, 2010. 